\title{
Mechanical hydraulic characteristic analysis scheme based on lightweight crowd data in mobile embedded devices
}

\author{
Shu-yi Guo* and Qi Si
}

\begin{abstract}
In order to improve the efficiency of mechanical and hydraulic control of the mechanical equipment, the analysis scheme of mechanical hydraulic characteristics based on lightweight crowd data was proposed in mobile embedded devices. Based on the mobile and embedded machinery equipment, a crowd lightweight data-driven analysis model is proposed to solve the hydraulic mechanical properties of nonlinear filtering with coarse-grained service detection. The engine of the mechanical equipment was connected with the hydraulic control module through the harmonic filter. Based on the output array of hydraulic characteristics and the transmission power of the mobile embedded node, the analysis scheme of mechanical hydraulic characteristics was proposed based on lightweight crowd data in mobile embedded devices. Based on the experiment evaluation result, the hydraulic analysis performance and mechanical equipment support ability of the proposed scheme is better than the static node scheme.
\end{abstract}

Keywords: Mechanical hydraulic characteristics, Embedded crowd systems, Lightweight crowd data, Mechanical hydraulic service driven

\section{Introduction}

Hydraulic control technology of mechanical equipment has been widely used in mechanical fault detection and robustness protection and so on [1]. How to determine the mechanical hydraulic mechanical transmission system in the structure of the detection scheme [2]? According to the mechanical condition, setting the parameters of mechanical hydraulic control system and antenna matching is one of the key problems. In the design of mechanical hydraulic control module and the detection node, how to combine the mechanical speed and mechanical equipment working load effectively becomes the main factor that restricts the mechanical power and the economy [3].

In view of the above problems, a series of research results are obtained. Pattison et al. [4] reformulated a pyroclastic model to recover hydraulic conductivity with more appropriate fluid-flow boundary conditions. Using an online monitoring system, tests were carried out in

\footnotetext{
* Correspondence: shyiguo@sina.com

North China University of Water Resources and Electric Power, Zhengzhou 450011, China
}

article [5], which could be used to measure the mechanical characteristics of a hydraulic high-voltage circuit breaker. The performance of hydraulic excavator with pump and valve was researched in article [6], which has been combined separate meter in and meter out circuits. The metaphysics-coupled modeling was proposed and simulated by Bing et al. [7], which has been used in the hydraulic-operating mechanism for a SF6 high-voltage circuit breaker.

The author of article [8] provided an in-depth look into how the systems have- or will be changed. An estimator based on the iterated unscented Kalman filter algorithm was proposed to identify model parameters in article [9]. The author in article [10] presented a fast method for calculating energy savings that would result from installing variable-frequency-drives on the seawater cooling pumps on ships. The distributed generation system managed was proposed by a power converter with reduced number of components [11]. The authors of article [12] studied the sensor hysteresis modeling with the Preisach model and did the regression analysis with support vector machine. 
However, the above research results ignored the relationship of mechanical hydraulic characteristics and system execution efficiency. We proposed the analysis scheme of mechanical hydraulic characteristics based on lightweight crowd data in mobile embedded devices.

\section{Lightweight crowd data-driven model}

On the analysis network of mobile machinery equipment, we will simplify the machinery and equipment with mobility and embedded technology to solve the problems of hydraulic mechanical properties of nonlinear filtering of coarse-grained service detection.

A crowd lightweight data-driven analysis model with fine-grained characteristics is proposed. The model includes the control protocol of embedded nodes in machinery equipment, the elimination mechanism of interference, the control protocol of transmission power, and the crowd data-driven mechanism.

We assume that the mobile embedded nodes are deployed in a mechanical device with a hop detection signal within the effective sensing radius. The node can sense the hydraulic real-time state of the mechanical equipment. In the characteristic analysis network of the dynamic mechanical equipment, the signal transmission power of the mobile embedded node can be adjusted adaptively according to the size of the crowd data. The effective sensing radius of the mobile embedded node is related to the residual energy of the node. Mobile embedded node adjusts its position and signal transmission power according to the residual energy. When the embedded node forwarding of swarm intelligence data meets the demand characteristic analysis of mechanical hydraulic, adjusting mobile embedded nodes of the perceived distance and signal transmit power, at the edge of the state, achieves lightweight data-driven service push.

Moving track of mobile embedded node is shown in Fig. 1. Among them, there are three kinds of critical points in mobile embedded node. The dotted circle in the graph is the effective sensing radius of the embedded node. When the residual energy of mobile embedded nodes is decreased and the crowd data is larger, the node can move from $\mathrm{A}$ to $\mathrm{B}$ in order to meet the requirement of the mechanical hydraulic characteristic detection. In order to achieve lightweight data-driven, the data size is reduced from the $\mathrm{B}$ position to the $\mathrm{C}$ position. Among them, A, B, C three positions can be driven by the crowd data to achieve adaptive switching.

Crowd data scale could be obtained by formula (1). Here, $S_{\mathrm{MC}}$ is the crowd data scale. $D_{i}$ is the mobile embedded device crowd forwarding data. $\bar{D}_{\mathrm{MN}}$ is the average value of the hydraulic characteristics of the data in $\mathrm{A}, \mathrm{B}, \mathrm{C}$ three different locations. $D_{\mathrm{MC}}$ is the total data of crowd sensing. $I_{\mathrm{MN}}$ is the set of mobile crowd nodes. $I_{\mathrm{ME}}$ is the set of mechanical equipment.

$$
\frac{S_{\mathrm{MC}}=\sum_{i \in I_{\mathrm{MN}}}\left(D_{i}-\bar{D}_{\mathrm{MN}}\right)}{\sqrt{\sum_{i \in I_{\mathrm{MN}} \cap I_{\mathrm{ME}}}\left(D_{\mathrm{MC}}\right)}}
$$

The transfer process of the three locations is as follows:

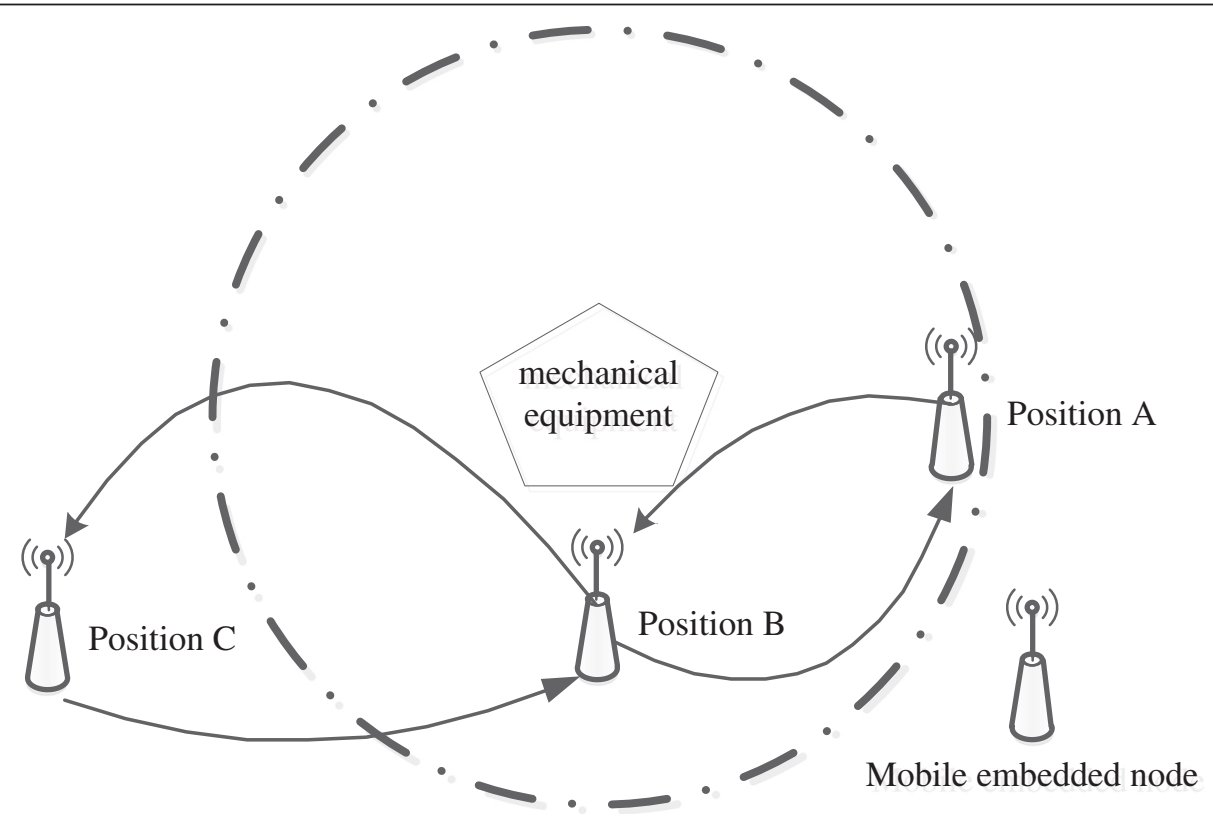

Fig. 1 Moving track of mobile embedded node for mechanical equipment 


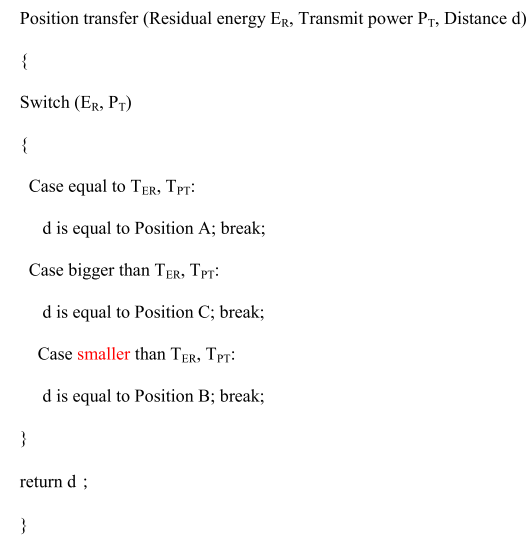

Here, $T_{\mathrm{ER}}$ denotes the residual energy threshold. $T_{\mathrm{PT}}$ denotes the signal transmit power threshold. These values could be obtained by formula (2).

$$
\left\{\begin{aligned}
T_{\mathrm{ER}}= & \frac{\sum_{i=1} E_{i} \cdot S_{i}(d)}{M_{N}} \\
T_{\mathrm{PT}}= & \frac{\sum_{i=1} T_{i} \cdot D_{i}(d)}{D_{\mathrm{ME}}}
\end{aligned}\right.
$$

Here, $E_{i}$ is the energy consumption of mobile embedded node. $S_{i}$ is the crowd data. $M_{N}$ is the number of mobile crowd nodes. $T_{i}$ is the signal sending power of mobile crowd node. $D_{i}$ is the sending data of mobile embedded node. $D_{\mathrm{ME}}$ is the hydraulic characteristic data of mechanical equipment.

For a number of mechanical equipment at the same time to detect and analyze the hydraulic characteristics, there is a need to set up a mobile mechanical equipment characteristic analysis network. The network is composed of multiple mobile embedded nodes. Each node has the crowd function. Topology is shown in Fig. 2.

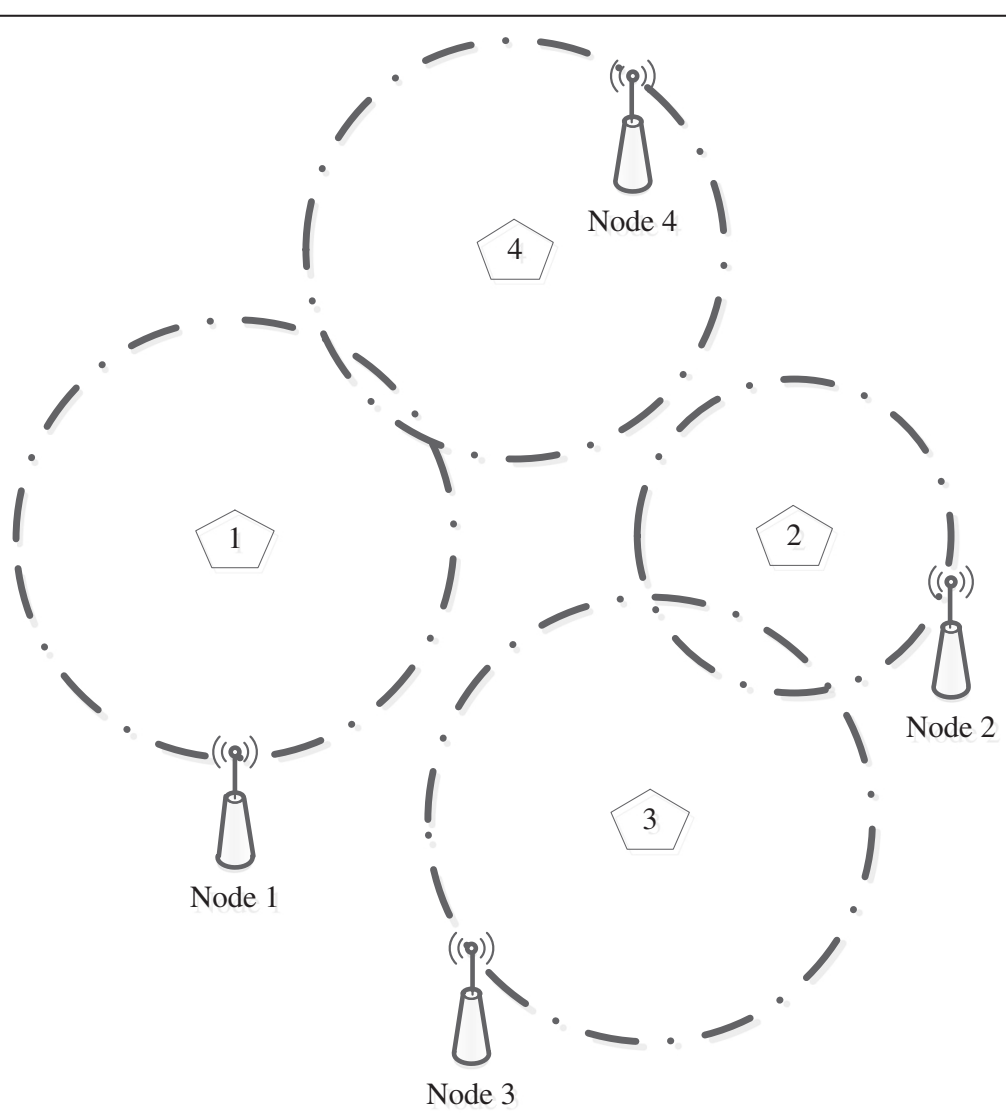

Fig. 2 Mobile mechanical equipment characteristic analysis network 
When the node 3 is unable to detect and analyze the hydraulic characteristics of the mechanical equipment due to energy failure, the nodes are 1,2 , and 4 after moving to update the analysis network topology, as shown in Fig. 3. The solid circle for 1 node represents the analysis of network characteristics of hydraulic machinery and equipment 2 and 4.

\section{Mobile embedded machine hydraulic characteristic analysis mechanism}

The data output and input of the hydraulic control module in the mechanical equipment must have a linear proportional relationship. The linear relation can be realized by the adaptive controller of embedded node. When a mechanical hydraulic control signal is sent to be sent, the mobile embedded node moves to the mechanical equipment end of the hydraulic valve radio frequency working area on the circumference. Hydraulic pump piston $\mathrm{B}$ chamber suctions air, and at the same time, $\mathrm{C}$ cavity starts frequency division antenna to transmit hydraulic signal. When the piston of the hydraulic pump is discharged from the D cavity, the feedback signal of the mobile embedded node is received, and the balance ratio of the elastic and electromagnetic coupling is controlled. Based on the force of the piston B cavity and the $\mathrm{C}$ cavity, combined with the pressure of the $\mathrm{D}$ chamber, the hydraulic characteristic analyses of the mechanical equipment and the control of the hydraulic pump are completed, as shown in Fig. 4. The hydraulic control module is used to separate and fuse the cavities B, C, and $\mathrm{D}$. The data transmission is deployed in the mobile embedded node with antenna.

The engine of the mechanical equipment is connected with the hydraulic control module through the harmonic filter. Formula (3) shows the relationship between the output of the hydraulic characteristics and the transmission power of the mobile embedded nodes.

$$
\frac{P_{\text {sending }}=\Delta v_{\text {sending }} A_{\mathrm{HC}}}{2500 \sin \alpha}
$$

Here, $P_{\text {sending }}$ is the transmission power, $v_{\text {sending }}$ is the transmission rate, and $A_{\mathrm{HC}}$ is the output of the hydraulic characteristics.

In order to improve the efficiency of the mechanical hydraulic transmission, we select the hydraulic control target value as the maximum power load. In order to ensure the stability and safety of the mechanical hydraulic module, the working efficiency of the cavity $B_{\mathrm{CD}}$ is always maintained at the maximum power $P_{\max }$, such as formula (4). In order to ensure the validity and credibility of the analysis of hydraulic characteristics, the target value of the antenna array of the hydraulic control system and the mobile embedded node must meet formula (5).

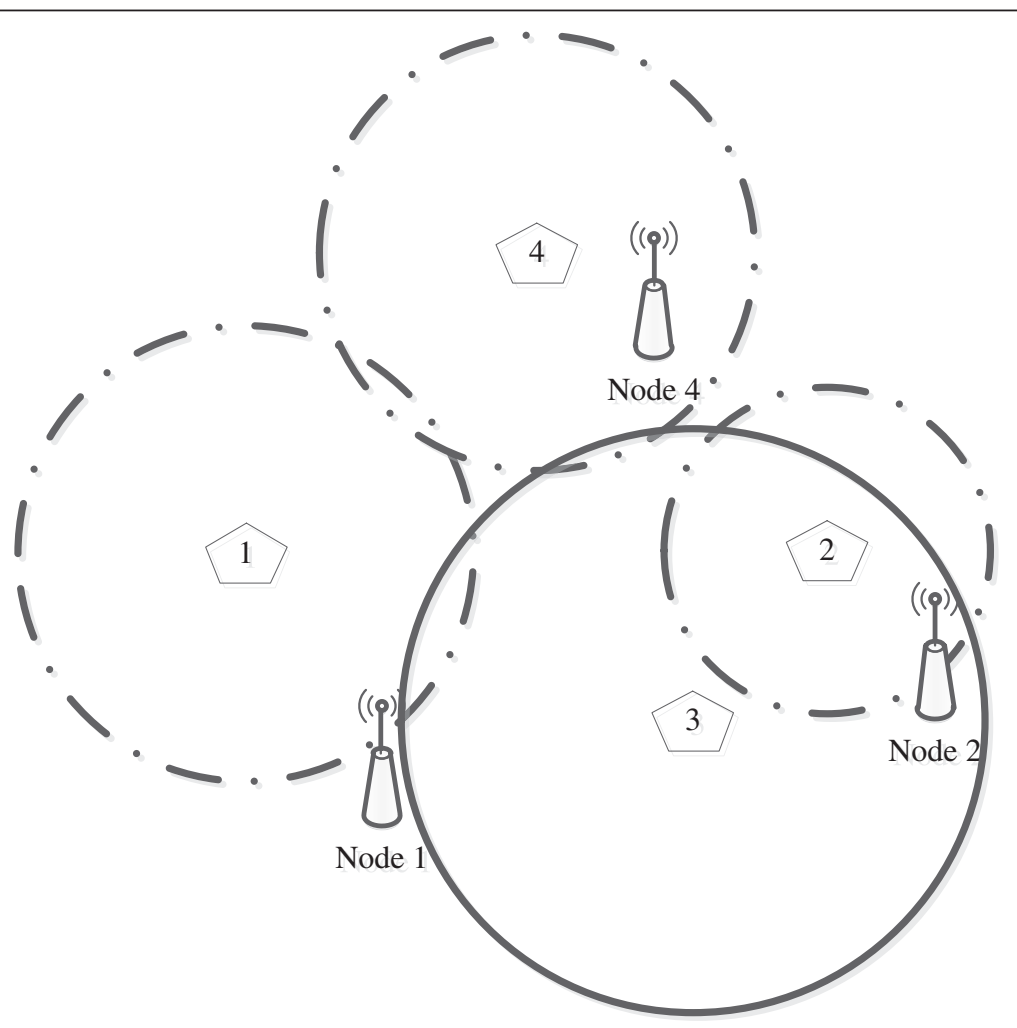

Fig. 3 Perceptual topology of hydraulic feature data based on mobile crowd 


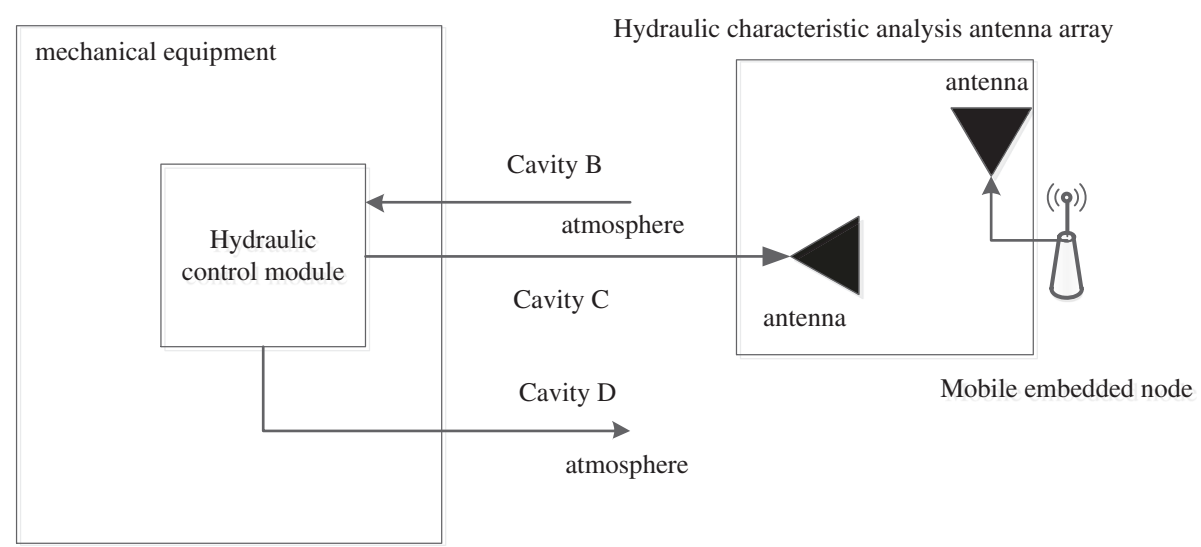

Fig. 4 Architecture of hydraulic characteristic analysis of mechanical equipment and hydraulic pump control

$$
\frac{P_{\max }=2500 \eta_{\mathrm{MN}} \sin \alpha}{\Delta \delta_{\mathrm{ME}}}
$$

Here, $\eta_{\mathrm{MN}}$ is the working efficiency of mobile embedded node. $\delta_{\mathrm{ME}}$ is the working efficiency of mechanical equipment.

$$
\left\{E_{\mathrm{ME}}=\int_{t_{1}}^{t_{2}} S_{\mathrm{HC}}\left(\frac{\cos \alpha}{\eta_{\mathrm{ME}}}\right) d t A_{\mathrm{antenna}}=\frac{\Delta E_{\mathrm{ME}}}{2 \pi} \frac{d t}{d P_{\max }}\right.
$$

Here, $S_{\mathrm{HC}}$ represents the weight matrix of hydraulic analysis. $E_{\mathrm{ME}}$ represents the efficiency of the hydraulic control system. $A_{\text {antenna }}$ represents the antenna array, which can be obtained by differentiating the working time and the maximum power.

Hydraulic characteristics of the analysis mechanism to obtain the parameters of the hydraulic output torque $T_{\mathrm{HC}}$, hydraulic control weight $W_{\mathrm{HC}}$, and mechanical hydraulic function area $S_{\mathrm{AHC}}$ are shown in formula (6).

$$
\left\{\begin{aligned}
T_{\mathrm{HC}} & =\frac{\sin (\alpha) E_{\mathrm{ME}}}{d \cdot \tan (\alpha-1)} \\
W_{\mathrm{HC}} & =\frac{\sin \left(\alpha_{A}-\eta\right)}{\sin \left(\alpha_{B}-\eta\right)}\left\|A_{\text {antenna }}\right\| \\
S A_{\mathrm{HC}} & =2 \pi \frac{d_{A}+d_{B}}{\sqrt{d_{A} d_{B}}} v_{\text {sending }}
\end{aligned}\right.
$$

\section{Performance analysis and verification}

In this paper, based on the lightweight crowd datadriven, this paper presents the analysis mechanism of the mobile embedded machine hydraulic characteristics. In order to analyze and verify the performance of the proposed mechanism, we researched the hydraulic characteristics of the mechanical hydraulic drive system. The mechanical parameters of the drive system are shown in
Table 1. Mobile embedded node parameters are set as shown in Table 2. The experimental environment is a closed indoor environment for avoiding the external noise interference. We mainly analyze the mechanical performance of the mechanical equipment in the normal working line.

In first experiment, the mobile embedded nodes were deployed in a set of transmission system. The hydraulic analysis performance of the hydraulic drive system is compared with that of the horizontal coordinate.

We studied the three sets of transmission system in the second experiment. The analysis network was composed of three mobile embedded nodes, for studying the hydraulic monitoring time and natural stop transfer ratio, which record the pressure characteristics of the hydraulic system to carry out a comparative analysis of the situation.

The results of the first experiment are shown in Fig. 5. The mechanical hydraulic performance analysis of static and dynamic nodes is compared. The horizontal coordinate indicates the working load ratio of the hydraulic transmission system, from 10 to $90 \%$. The vertical coordinate is the mechanical hydraulic output torque.

\begin{tabular}{|c|c|c|c|}
\hline Parameters & Value & $\begin{array}{l}\text { Descriptions } \\
\text { of system }\end{array}$ & Value \\
\hline $\begin{array}{l}\text { Weight of mechanical } \\
\text { hydraulic transmission } \\
\text { system }\end{array}$ & $\begin{array}{l}15,000 \\
(\mathrm{~kg})\end{array}$ & Speed of transfer & $2500 \mathrm{rpm}$ \\
\hline Driving power & $\begin{array}{l}145 \\
(\mathrm{~kW})\end{array}$ & System initialization delay & $2.5 \mathrm{~min}$ \\
\hline Air flow rate & $\begin{array}{l}20 \\
\left(\mathrm{~m}^{3} / \mathrm{s}\right)\end{array}$ & Maximum bear weight & $2000 \mathrm{~kg}$ \\
\hline $\begin{array}{l}\text { Transmission } \\
\text { efficiency }\end{array}$ & $85 \%$ & $\begin{array}{l}\text { Evacuation time of } \\
\text { hydraulic pump }\end{array}$ & $10 \min$ \\
\hline $\begin{array}{l}\text { Transmission drag } \\
\text { coefficient }\end{array}$ & 0.04 & $\begin{array}{l}\text { Motor displacement of } \\
\text { hydraulic pump }\end{array}$ & $100 \mathrm{ml} / \mathrm{r}$ \\
\hline
\end{tabular}

Table 1 Parameter settings of mechanical hydraulic transmission system 
Table 2 Parameter settings of mobile embedded node

\begin{tabular}{llll}
\hline Parameters & Value & $\begin{array}{l}\text { Descriptions of } \\
\text { system }\end{array}$ & Value \\
\hline $\begin{array}{l}\text { Radio frequency of } \\
\text { antenna }\end{array}$ & $2.4 \mathrm{GHz}$ & $\begin{array}{l}\text { Communication } \\
\text { protocol }\end{array}$ & IEEE \\
Transmission rate & $3002.11 \mathrm{n}$ \\
Working power & $5 \mathrm{~V}$ & Number of antenna & 3 \\
Weight & $0.8 \mathrm{~kg}$ & Dattery capacity & $5200 \mathrm{mAh}$ \\
\hline
\end{tabular}

From Fig. 5, it is found that with the increase of working load, the static node is difficult to analyze the hydraulic output torque. When the working load ratio is greater than $40 \%$, the output torque remains the same. When the work load ratio is greater than $70 \%$, there is a sudden drop, resulting in a large error analysis. The conclusion is not reliable. Because of the dynamic node, according to its own residual energy and output power, the three-point positioning switch, a full range of mechanical equipment to monitor the hydraulic output characteristics, and real-time statistical hydraulic output torque have a strong credibility.

The results of the second experiment are shown in Figs. 6 and 7. In Fig. 6, the horizontal coordinate is monitoring time hydraulic. The longitudinal coordinate is the position tracking error. Figure 6 compares the embedded mobile node tracking position error of hydraulic analysis algorithm with crowd lightweight data-driven algorithm (HA-CLDD) and hydraulic analysis algorithm with data-driven (HA-DD) based on lightweight crowd data-driven hydraulic analysis. Lightweight data-driven hydraulic analysis algorithm could provide the performance guarantee of the long time monitoring. Based on the duty cycle and the working load of the mechanical hydraulic pump, the lightweight crowd data-driven

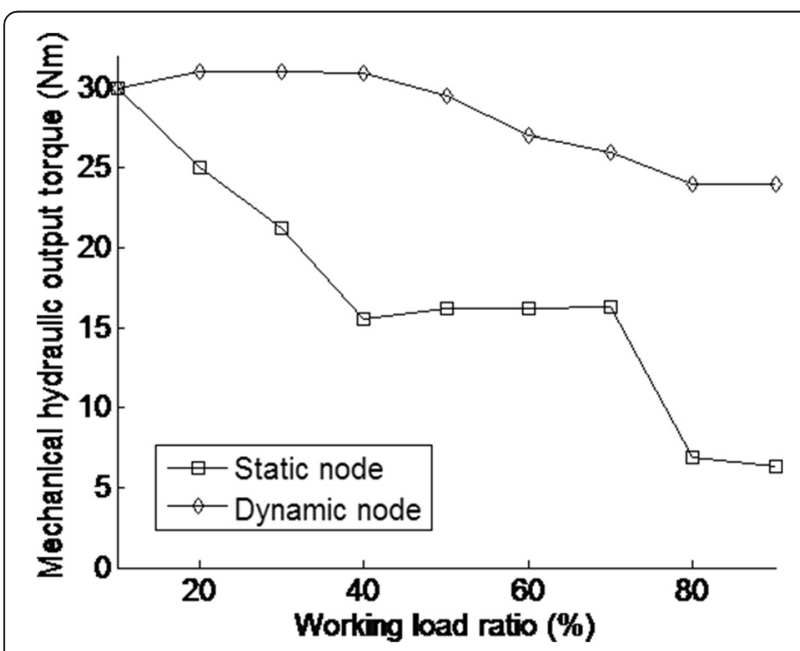

Fig. 5 Mechanical hydraulic output torque

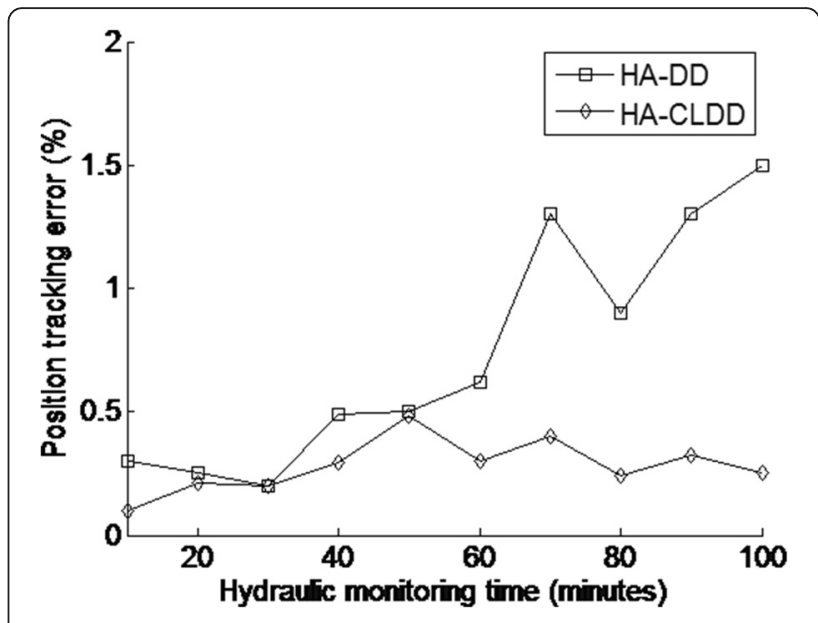

Fig. 6 Position tracking error

scheme could adjust the efficiency of the system to provide the accurate RF information for mobile embedded nodes.

$Y$-axis of Fig. 7 is a natural stop ratio. Figure 7 gives the comparison analytical results of the natural stop ratio based on the mechanical and hydraulic properties of embedded mobile node with mechanical hydraulic characteristic analysis (W-MHCA) and hydraulic characteristic analysis of mechanical transmission system (WO-MHCA). We completed a 100-min test of mechanical transmission system.

Through hydraulic machinery characteristic analysis of embedded mobile node, the situation of hydraulic transmission system could be accurately predicted. The cavities B, C, and D would be adaptively adjusted. The transmission system always maintain the best working condition based on the above issues, which would improve work efficiency and load capacity.

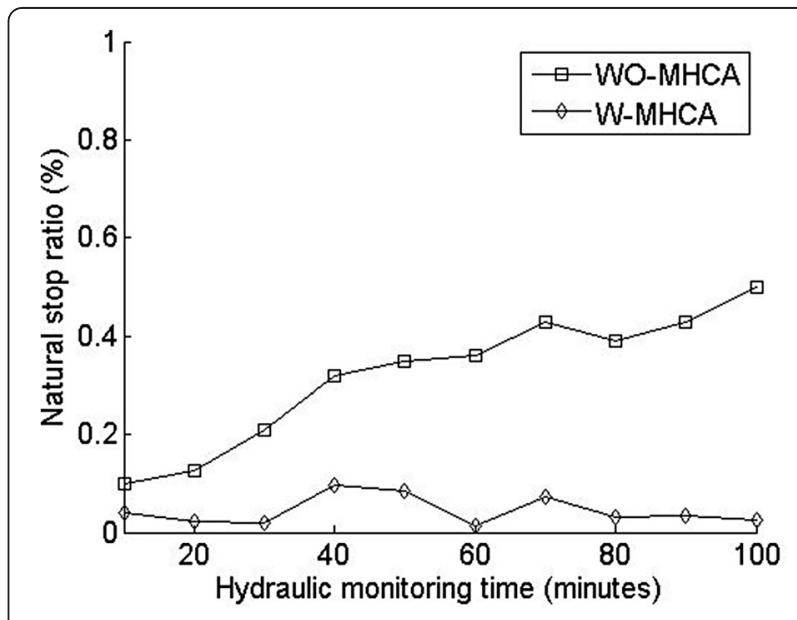

Fig. 7 Natural stop ratio 


\section{Conclusions}

The analysis scheme of mechanical hydraulic characteristics was proposed based on lightweight crowd data in mobile embedded devices to solve the mechanical hydraulic equipment control and improve the efficiency of mechanical work. Firstly, a population intellectual lightweight data-driven analysis model was proposed with the mobile embedded machinery and equipment, which was used to solve the hydraulic mechanical properties of nonlinear filtering of coarse-grained service detection. Secondly, we studied the engine of the mechanical equipment with the hydraulic control module through the harmonic filter. Then, we designed the mobile embedded node by considering the output array of hydraulic characteristics and the transmission power. Based on the above basis, the analysis scheme of mechanical hydraulic characteristics was proposed based on lightweight crowd data in mobile embedded devices. Based on the experiment evaluation result, the hydraulic analysis performance and mechanical equipment support ability of the proposed scheme is better than the static node scheme and without analysis state.

\section{Competing interests}

The authors declare that they have no competing interests.

Received: 19 May 2016 Accepted: 8 August 2016

Published online: 22 August 2016

\section{References}

1. OH Souza, N Barbieri, AHM Santos, Study of hydraulic transients in hydropower plants through simulation of nonlinear model of penstock and hydraulic turbine model [J]. IEEE Trans. Power Syst. 14(4), 1269-1272 (1999)

2. W Liao, S Chen, C Chen et al., Research of negative flow control characteristics for axial piston pump based on hydraulic and mechanical co-simulation[C]// International Conference on System Science, Engineering Design and Manufacturing Informatization. Chengdu, China, (IEEE Press, 2012), pp. 79-83

3. M Noulhiane, S Samson, S Clémenceau et al., High-accuracy range detection radar sensor for hydraulic cylinders [J]. Sensors J. IEEE 14(3), 734-746 (2014)

4. AJ Pattison, M Mcgarry, JB Weaver et al., Spatially-resolved hydraulic conductivity estimation via poroelastic magnetic resonance elastography [J]. IEEE Trans. Med. Imaging 33(6), 1373-80 (2014)

5. C Wang, C Liu, Experimental study of the online monitoring of the mechanical characteristics of a hydraulic high-voltage circuit breaker[C]// International Conference on Electric Utility Deregulation and Restructuring and Power Technologies. Changsha, China, (IEEE Press, 2015), pp. 2706-09

6. $\mathrm{L}$ Ge, Z Dong, W Huang et al., Research on the performance of hydraulic excavator with pump and valve combined separate meter in and meter out circuits[C]// International Conference on Fluid Power and Mechatronics. Harbin, China, (IEEE Press, 2015), pp. 37-41

7. B Xu, R Ding, J Zhang et al., Multiphysics-Coupled Modeling: Simulation of the Hydraulic-Operating Mechanism for a SF6 High-Voltage Circuit Breaker[J]. IEEE/ASME Trans. Mechatron. 21, 379-93 (2016)

8. B Sarlioglu, CT Morris, More electric aircraft: review, challenges, and opportunities for commercial transport aircraft [J]. IEEE Trans. Transportation Electrification 1(1), 54-64 (2015)

9. H Huang, M Yang, W Zang et al., In vitro identification of four-element windkessel models based on iterated unscented Kalman filter [J]. IEEE Trans. Biomed. Eng. 58(9), 2672-80 (2011)
10. frequency-drive applications on ship central cooling systems [J]. IEEE Trans. Ind. Appl. 50(50), 1286-1294 (2014)

11. ECD Santos, CB Jacobina, N Rocha et al., Single-phase to three-phase fourleg converter applied to distributed generation system [J]. IET Power Electron. 3(6), 892-903 (2010)

12. C Yang, C Li, The application of support vector machine in the hysteresis modeling of silicon pressure sensor [J]. IEEE Sensors J. 11(9), 2022-2026 (2011)

\section{Submit your manuscript to a SpringerOpen ${ }^{\circ}$ journal and benefit from:}

- Convenient online submission

- Rigorous peer review

- Immediate publication on acceptance

- Open access: articles freely available online

- High visibility within the field

- Retaining the copyright to your article

Submit your next manuscript at $>$ springeropen.com 